\title{
Painful mechanical stimulation evokes activation of distinct functional areas in the brain: comparison of normal subjects and two patients with neuropathic pain
}

\author{
Tatsunori Ikemoto ${ }^{1}$, Takahiro Ushida ${ }^{1}$, Shigeki Tanaka ${ }^{4}$, \\ Kazuo Morio ${ }^{2}$, Vadim S. Zinchuk ${ }^{3}$, Toshikazu Tani ${ }^{1}$, \\ Shinichirou Taniguchi ${ }^{1}$, and Akio Ushida ${ }^{5}$
}

\author{
${ }^{1}$ Department of Orthopaedic Surgery, Kochi Medical School, Kochi 783-8505, Japan \\ ${ }^{2}$ Department of Radiology, Kochi Medical School, Kochi 783-8505, Japan \\ ${ }^{3}$ Department of Anatomy and Cell Biology, Kochi Medical School, Kochi 783-8505, Japan \\ ${ }^{4}$ Department of Psychology, Jin-Ai University, Fukui 915-0015, Japan \\ ${ }^{5}$ Department of Electrical and Electronic Engineering, Faculty of Engineering, \\ Tokushima University, Tokushima 770-8506, Japan
}

[Received 28 April 2003, Accepted 3 July 2003]

\begin{abstract}
We employed functional magneto-resonance imaging to compare the pattern of brain activation evoked by painful and non-painful mechanical stimulation in normal volunteers and patients with neuropathic pain. Stimulation was performed using von Frey filaments. In normal volunteers, painful stimulation caused significant activation in the inferior parietal lobule (area 40), primary motor area (area 4), cingulate gyrus (area 24/31), middle temporal Gyrus (area 21/37), thalamus, and cerebellum. In the non-pain condition, SI, parietal lobule and frontal lobe were activated. In the patients, stimulation in the area of allodynia, in the non-pain condition elicited painful feelings and caused a wider area of activity when compared to the area of activity when the palm of unaffected side was stimulated. The activation was promoted in the frontal and occipital lobes, cingulate gyrus, and cerebellum. These findings suggest that neuropathic pain patients exhibit differences in the pain perception that may reflect chronical activation of the appropriate functional brain areas.
\end{abstract}

Key words: fMRI, pain perception, allodynia, brain imaging

\section{INTRODUCTION}

Recent advances in neuroimaging, such as wide use of positron emission tomography (PET) and functional magneto-resonance imaging (fMRI), have improved our understanding of the mechanisms of pain perception in the brain. FMRI is able to detect neurological activation in response to tasks by revealing BOLD (Blood Oxygen Level Dependent) signals in selected areas of the brain. BOLD-fMRI produces images with a temporal resolution at the order of 100 milliseconds and a spatial resolution much greater 
than that of PET and SPECT scanning. This implies the potential ability to image transient cognitive events more reliably and an opportunity to distinguish smaller structures. Moreover, in contrast to PET and SPECT, fMRI is noninvasive technique and does not require an injection of radioactive materials so that the same subject can be imaged a number of times. The latter allows following serial changes in the brain easier. This is reflected in numerous studies conducted on normal volunteers (Talbot et al., 1991; Casey et al., 1994; Hsieh et al., 1994; Svensson et al., 1997; Iadarola et al., 1998; Coghill et al., 1994; Baron et al., 1999). It was found that in response to noxious pain stimulation activation occurs in the primary somatosensory cortex, thalamus, insula, and cingulate gyrus (Casey et al., 1994; Svensson et al., 1997; Iadarola et al., 1998; Coghill et al., 1994). It was also reported that patients with permanent pain syndromes experience increased sensitivity to pain. Repeated "pain experiences" may not simply cause an increase in the magnitude of pain perceived, but also affect reception and emotion associated with it (Melzack, 1980; Ploghaus et al., 2001; Reiman et al., 1989). These findings suggest that in pain patients changes occur not only in the primary somatosensory cortex, but also in the limbic system and other brain regions involved in memory and emotion.

Since pain itself is subjective and its etiology among various patients differs (Dickens et al., 2002; Farrar et al., 2001; Dalton and McNaull, 1998), objective evaluation of the pain with the help of standardized methods is hardly reliable. However, as the final destination of interpretation of pain is the cerebrum, a certain degree of objective evaluation of pain should be possible by comparing the regions of excited brain between normal individuals and patients. In the present study, we employed simple and semi-quantitative mechanical stimulation with von Frey filaments, a routine tool used for sensory testing in clinics, to compare the regions of brain responsive to pain in normal volunteers and patients with allodynia. We attempted to evaluate what could be the effect of dynamic pain to the brain and how would it react to the chronic refractory pain.

\section{METHODS}

\section{Subjects}

Twelve normal volunteers (age range from 20 to 38 years, mean 27.6 years), 5 men and 7 women, and 2 patients with allodynia, a man and a woman, were enrolled in this study. All volunteers had no history of brain vascular diseases in the past. They were informed on the study purpose and gave a written consent. Patients with neuropathic pain were chosen among the patients with allodynia in the hand being treated in our clinic. One patient (Case I) was a 51-year-old woman with allodynia experienced after a cervical spinal cord injury and the other was a 54-year-old man (Case II) with complex regional pain syndrome type 1 (hereafter CRPS type 1). Both patients underwent routine MRI examination to exclude the possibility of brain vascular diseases. In normal volunteers, two different von Frey filaments were used: a noxious filament $490 \mathrm{mN}$ that gave a mechanically evoked pain by tapping, and an innocuous filament $11.5 \mathrm{mN}$ that gave only a touch sensation. The tapping stimulation was performed in the central recessed area of the right palm using a force that was sufficient to cause the filament to start to bend. In the neuropathic pain patients, the tapping stimulation was performed in the allodynia area and the same area on the contralateral side using the innocuous filament only.

\section{Stimulation protocol}

Prior to the beginning of the experiment patients were allowed to experience the intensity of pain using the filaments to be used in the tasks. The fMRI images were obtained in the supine position, with both palms facing up in a position that would allow the tasks to be supplied to the palm without any movement on the part of the subject. Over an interval of 336 seconds, several tasks were performed in block paradigms to compare the brain neurological activation in the stimulated versus unstimulated state.

In normal volunteers, the tapping stimulation was performed using the noxious filament or the innocuous filament in the central recessed area in the 
right palm using a force sufficient to cause the filament to start to bend. The evaluation of mechanically evoked pain with the noxious filament was performed in the subjects using the Visual analog scale (VAS): scale from 0 (no pain) to 10 (most intense pain imaginable). The task with each filament was a tapping stimulation of 16 seconds, and the rate of tapping matched the $3.5 /$ second rhythm that is generated during imaging. During imaging, the tasks were performed six times, three times with the noxious filament and three times with the innocuous filament; the subjects were not informed on the order of the tasks.

In patients with neuropathic pain, it was known prior to the beginning of the experiment that even few stimulations with the innocuous filament cause a great deal of pain and that the provoked pain persists for some time. Thus, the tapping stimulation was performed five times with 2-seconds intervals. Stimulation by the innocuous filament in the allodynia area was evaluated using VAS. Stimulation of the unaffected area was conducted in the same way as stimulation of the affected side. This task was performed at $38 \mathrm{sec}$ intervals, 4 times per side.

\section{fMRI procedure}

fMRI imaging was performed for the entire brain using GE Co. apparatus, SIGNA 1.5 Teslar, blood oxygenation level dependent (BOLD), T2 weighted multislice gradient EPI sequence $(\mathrm{TE}=40 \mathrm{~ms}, \mathrm{TR}=$ $4000 \mathrm{~ms}$, flip angle $=90$ degrees), slice width $7 \mathrm{~mm}$, gap $1 \mathrm{~mm}, 17$ slices. The subjects were imaged in the supine position in the standard manner, with a sponge placed under the lower half of the head and fixed with tape to minimize artifacts due to movement. The subjects were instructed to close their eyes during imaging. Noise generated during the imaging was suppressed with earplugs.

\section{Image analysis}

Image analysis was performed on a Unix workstation using SPM99 software (Wellcome Department of Cognitive Neurology, Institute of Neurology, London http://www.fil.ion.ucl.ac.uk/spm) (Frinston et al. 1995).
fMRI images were transferred to a Unix workstation to obtain the format suitable for analysis. All formatted volumes were analyzed using SPM99. Images were realigned, spatially normalized to a standard EPI template and finally smoothed using a Gaussian kernal of $8 \mathrm{~mm}$ (Friston, 2002; Price and Friston, 1997; Friston et al., 1995; Friston et al., 1994). Results for volunteer group were modeled using a boxcar design convolved with a haemodynamic response function chosen to represent the relationship between neuronal activation and blood flow changes. The boxcar had three conditions, namely noxious stimuli, innocuous stimuli, and rest. Signal changes related to noxious tapping task or innocuous tapping task were obtained. Significance was determined on a voxel-by-voxel basis using $\mathrm{t}$ statistics, which was then transformed into a normal distribution. Active locations were expressed in terms of $\mathrm{x}, \mathrm{y}, \mathrm{z}$ coordinates converted to Talairach space (Talairach and Tournoux, 1988). Cluster of voxels which had an uncorrected threshold $\mathrm{p}<0.001$ were considered as showing significant differences. The same analytical procedure was used for individual analysis of each neuropathic pain case.

\section{RESULTS}

Activation of the brain was studied in 12 normal subjects in response to mechanically evoked pain and touch sensation stimuli. We observed wide variations among them for both the mechanically evoked pain and touch sensation stimulation. Thus, the normals were grouped for each task, and the mean was obtained for all of them. The pain stimulus from noxious filament gave a mean VAS of 4.2 and caused an increased activation in the contralateral inferior parietal lobule (area 40), primary motor area (area 4), cingulate gyrus (area 24/31), middle temporal gyrus (area 21/37), insula, thalamus, cerebellum, and in the ipsilateral inferior parietal lobule (area 40), temporal lobe (area 42) thalamus, cerebellum (Table 1, Fig. 1-A). Touch sensation stimulus by the innocuous filament gave neurological activation in the contralateral postcentral gyrus SI (area 1), parietal lobe (area 40) and bilateral frontal lobe (area 9/44, 46) (Table 1, Fig. 1-B). 
Table 1 Brain areas activated by noxious and innocuous mechanical stimulation in normal volunteers

Anatomical reigion

Noxious Stimulation to Rt. palm

Contralateral (=Lt. Hemishere)

Inferior Parietal Lobule (BA 40)

Frontal Lobe, Motor Cortex (BA 4)

Thalamus

Cingulate Gyrus (BA 24/31)

Middle Temporal Gyrus (BA 21/37)

Insula

Ipsilateral (=Rt. Hemishere)

Inferior Parietal Lobule (BA 40)

Supramargnal Gyrus

Temporal Lobe (BA 42)

Thalamus

Cerebellum Vermis

Cerebellum Posterior Lobe

Cerebellum Posterior Lobe

Innocuous (only tactile) Stimulation to Rt. palm

Contralateral

Postcentral Gyrus, SI (BA 1)

Ipsilateral

Parietal Lobe, Postcentral Gyrus (BA 40)

Frontal Lobe (BA 46)

Frontal Lobe (BA 9/44)

uncorrected threshold $\mathrm{p}<0.001$

A
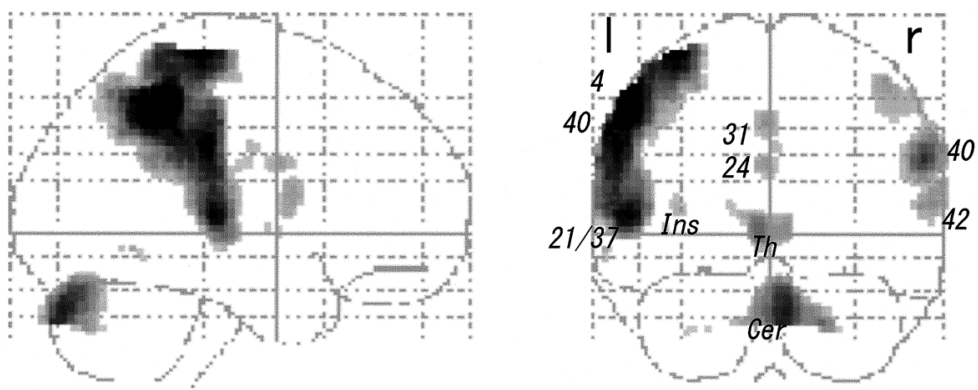

B
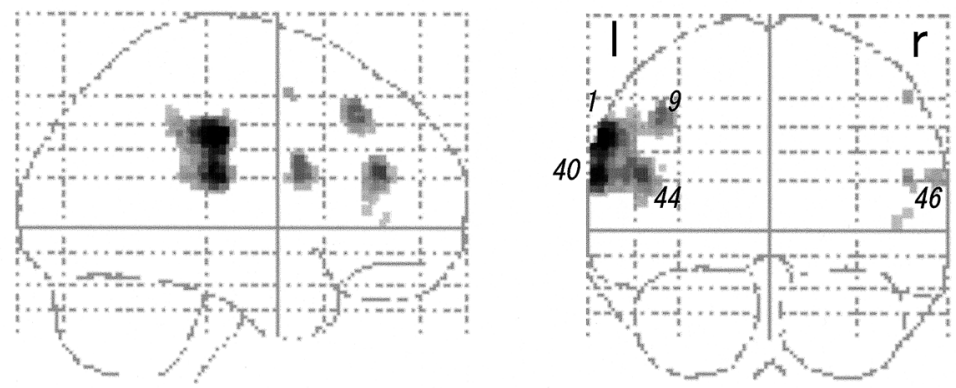

Fig. 1 Statistical maps of regions with mean Blood Oxygenation Level Dependent (BOLD) signals during noxious and innocuous mechanical tapping stimulation of the right palm in normal volunteers. Wide areas of inferior parietal lobule (area 40), primary motor area (area 4), cingulate gyrus (area 24), thalamus, and cerebellum strongly responded to noxious mechanical tapping $(\mathbf{A})(\mathrm{p}<0.001)$. Innocuous tapping elicited limited activation in the sensory cortex and the frontal lobe only $(\mathbf{B})(\mathrm{p}<0.001)$. Arabic numerals indicate Brodmann's areas. Ins: insula, Th: thalamus, Cer: cerebellum. 
Table 2 Brain areas activated by mechanical stimulation using innocuous filament in patients with neuropathic pain

\begin{tabular}{ll}
\hline Anatomical reigion & \\
\hline Case I: & \\
Neuropathic palm (Rt. palm) stimulation & \\
Contralateral (=Lt. Hemishere) & Ipsilateral (=Rt. Hemishere) \\
$\quad$ Frontal Lobe, Suppl. Motor Area (BA 6) & Occipital Lobe, Lingual Gyrus (BA 17) \\
Occipital Lobe, Lingual Gyrus (BA 18) & Occipital Lobe, Cuneus (BA 19) \\
$\quad$ Posterior Cingulate (BA 23) & Posterior Cingulate (BA 31) \\
& Cerebellum Posterior Lobe \\
Sound palm (Lt. palm) stimulation & \\
Contralateral (=Rt. Hemishere) & Ipsilateral (=Lt. Hemishere) \\
Cerebellum Anterior Lobe & Frontal Lobe (BA 10) \\
Cerebellum Posterior Lobe & \\
\hline Case II: & \\
Neuropathic palm (Rt. palm) stimulation & \\
Contralateral (=Lt. Hemishere) & \\
Frontal Lobe & Ipsilateral (=Rt. Hemishere) \\
Parietal Lobe, Precuneus & Superior Parietal Lobe (BA 5) \\
Supramarginal Gyrus (BA 40) & Parietal Lobe, Precuneus (BA 7) \\
Cingulate Gyrus & Occipital Lobe, Cuneus (BA 17) \\
Cerebellum Posterior Lobe & Cingulate Gyrus (BA 31) \\
Sound palm (Lt. palm) stimulation & Cerebellum Posterior Lobe \\
No activation was evoked & \\
uncorrected threshold p<0.005 & \\
\hline
\end{tabular}

Stimulus with the innocuous filament in the allodynia area of the patient with neuropathic pain after a cervical spinal cord injury (Case I) resulted in pain with the VAS of 6.1. This stimulus was used as a task, and fMRI showed significantly increased neurological activation in the contralateral supplementary motor area (area 6), occipital lobe (area 18), posterior cingulate (area 23) and in the ipsilateral occipital lobe (area 17/19), posterior cingulate (area 31), cerebellum (Table 2, Fig. 2-C). Similar stimulus to the unaffected area on the contralateral palm caused significant promotion of neurological activation only in the frontal lobe (area 10) and the cerebellum (Table 2, Fig. 2-D).

In the patient with CRPS type 1 (Case II), stimulus elicited by the innocuous filament in the allodynia area caused pain with a VAS of 10. This stimulus was used as a task, and fMRI showed significant promotion of neurological activation in the contralateral inferior parietal lobule (area 40), frontal lobe, occipital lobe, cingulate gyrus, cerebellum in the ipsilateral superior parietal lobe SII (area 5), parietal lobe percuneus (area 7), occipital lobe cuneus (area 17), posterior cingulate (area 31), cerebellum (Table 2, Fig. 2-E). Similar stimulus of the unaffected area on the contralateral palm caused no significant activation (Table 2, Fig. 2F). In both patients, stimulation in the allodynia area caused a wider region of activity compared to the stimulation of the unaffected area as determined by V.O.I .

\section{DISCUSSION}

It is generally agreed that subjective factors play a critical role in the perception of pain. Therefore, evaluation of the pain intensity using questionnaires and VAS cannot be considered sufficiently objective. We attempted to evaluate the feeling of pain with a certain degree of objectivity by comparing the areas of brain responsive to pain in normal volunteers and in 

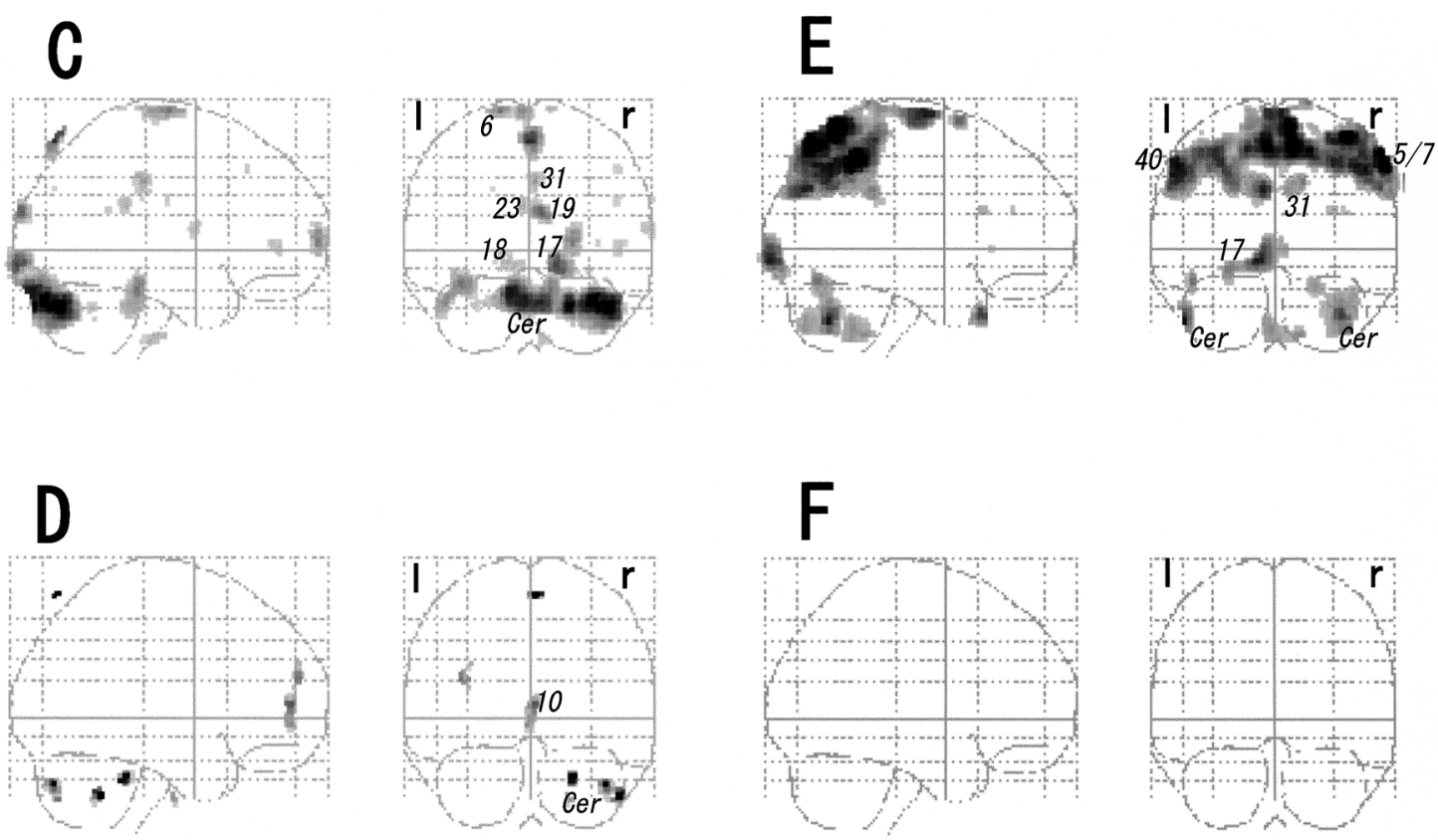

Fig. 2 In the patient with a cervical cord injury (case I) normally innocuous mechanical tapping stimulation of the painful right palm produced activation of suppl. motor area (area 6), posterior cingulate (area 23/31), occipital lobe (17-19) and cerebellum $(\mathbf{C})(\mathrm{p}<0.005)$. Whereas, contralateral sound palm stimulation activated only frontal lobe (area 10) and cerebellum (D) $(\mathrm{p}<0.005)$. In the patient with CRPS type I, normally innocuous mechanical tapping stimulation of the painful right palm produced activation of inferior parietal lobule (area 40), superior parietal lobe S II (area 5), cingulated gyrus (area 31) and cerebellum $(\mathbf{E})(\mathrm{p}<0.005)$. No any elicited area was detected by mechanical stimulation of sound palm $(\mathbf{F})$ $(\mathrm{p}<0.005)$. Arabic numerals means Brodmann's areas. Cer: cerebellum.

patients with allodynia. We found differences between normal subjects in the patterns of promoted activation in response to innocuous and noxious stimulation. There was no evident relationship between the VAS and the extent of brain activation. However, in individual subjects, noxious stimulation resulted in activation of the wider region of the brain compared to innocuous stimulation in all subjects. Several studies have reported finding describing the areas of the brain activated by noxious pain stimulation in normals detected by PET and fMRI (Peyron et al., 2000). Our data showing activated regions, such as contralateral inferior parietal lobule (area 40), primary motor area (area 4), cingulate gyrus (area 24,31), insula, thalamus and cerebellum agree with these observations. Comparing to neuropathic pain patients, the innocuous filament tapping to the volunteers resulted in limited areas of activation. Although patients showed wider areas of activation by normally innocuous tapping of the allodynia site, we believe that these differences may be due to the fact that we used a stimulating filament that gave pain to patients with allodynia, but produced only slight stimulation to normals. That is, the used filament is extremely weak and is able to convey touch sensation stimulus to an extremely small area, so that activation occurs only in the limited areas of the brain.

In the patients with neuropathic pain, stimulation of the unaffected side with innocuous filament caused neurological activation of a small area, as was seen in normal volunteers. On the other hand, similar stimulation of the affected side that lasted for 2 seconds (5 times) to the affected side caused a wide neurological activation in areas that included cingulate gyrus and 
cerebellum. This activation was similar to the response seen to the mechanical pain stimulus with the noxious filament in the normals. This result suggests that neuropathic pain patients were hypersensitive to innocuous stimulation and that innocuous stimulation was perceived as pain. However, the sites and extent of neurological activation seen in the cingulate gyrus and thalamus differed. This suggests that neuropathic pain patients may have differences in the character of pain and other related changes due to the plasticity of the brain. It is worth mentioning results of the recent study reporting on the limitations of the measurements of BOLD phenomenon using fMRI (Yamamoto and Kato, 2002). This study indicated that such measurements may occasionally produce false-positive results when evaluating small ischemic areas of the brain. However, this does not seem to be of concern in our study, because we focused on the areas that were sufficiently large and have not used subjects with the history of cerebrovascular abnormalities.

We observed activation of cerebellum in response to noxious stimulation in normals and in response to stimulation of the areas of allodynia in patients with pain. Considering the involvement of cerebellum in pain, several reports were published on neurological activation to pain stimulation and visceral nociception (Saab and Willis, 2001; Iadarola et al., 1998; Svensson et al., 1997), but there is no consensus concerning the involvement of this region in pain. Further studies should clarify this issue.

\section{CONCLUSION}

We used von Frey filaments as the stimulus task to evaluate objective changes in activity of the regions of the brain in response to pain. It was found that patients with allodynia, as opposed to normal subjects, show differences in the perception of pain that may be explained by the chronical activation of the appropriate brain areas. The method described here should prove to be useful for determining the improvement of pain as a result of pharmacological intervention and for evaluation of the efficiency of used drugs.

\section{ACKNOWLEDGEMENT}

We thank Profs. H. Yamamoto, S. Yoshida, W. Ueda and Dr. D. Yoshida for scientific advice, Mss. Y. Yamagami, S. Sasaki and Mr. Kabasawa for technical assistance. This work was partially supported by the grants of Japanese Minsitry of Education, Science, Sports, Culture and Technology, Grant-in-Aid for Scientific Research (B), 14370467, 2002, and Academia Kochi grant, 2000.

\section{REFERENCES}

1) Baron, R., Baron, Y., Disbrow, E. and Roberts, T.P., Brain processing of capsaicin-induced secondary hyperalgesia: a functional MRI study. Neurology, 53 (1999) 548-557.

2) Casey, K.L., Minoshima, S., Berger, K.L., Koeppe, R.A., Morrow, T.J. and Frey, K.A., Positron emission tomographic analysis of cerebral structures activated specifically by repetitive noxious heat stimuli, J. Neurophysiol., 71 (1994) 802-807.

3) Coghill, R.C., Talbot, J.D., Evans, A.C., Meyer, E., Gjedde, A., Bushnell, M.C., et al., Distributed processing of pain and vibration by the human brain, $J$. Neurosci., 14 (1994) 4095-4108.

4) Dalton, J.A. and McNaull, F., A call for standardizing the clinical rating of pain intensity using a 0 to 10 rating scale, Cancer Nurs., 21 (1998) 46-49.

5) Dickens, C., Jayson, M. and Creed, F., Psychological correlates of pain behavior in patients with chronic low back pain, Psychosomatics, 43 (2002) 42-48.

6) Farrar, J.T., Young, J.P., Jr., LaMoreaux, L., Werth, J.L. and Poole, R.M., Clinical importance of changes in chronic pain intensity measured on an 11-point numerical pain rating scale, Pain, 94 (2001) 149-158.

7) Friston, K.J., Jezzard, P. and Turner, R., Analysis of Functional MRI Time-Series, Hum. Brain Mapp., 1 (1994) 153-171.

8) Friston, K.J., Ashburner, J., Poline, J.B., Frith, C.D., Heather, J.D. and Frackowiak, R.S., Spatial Registration and Normalization of Images, Hum. Brain Mapp., 2 (1995) 165-189.

9) Friston, K.J., Statistical Parametric Mapping. In: Human Brain Function II, Academic Press, 2002.

10) Hsieh, J.C., Hagermark, O., Stahle-Backdahl, M., Ericson, K., Eriksson, L., Stone-Elander, S., et al., Urge to scratch represented in the human cerebral cortex during itch, J. Neurophysiol., 72 (1994) 30043008 .

11) Iadarola, M.J., Berman, K.F., Zeffiro, T.A., ByasSmith, M.G., Gracely, R.H., Max, M.B., et al., 
Neural activation during acute capsaicin-evoked pain and allodynia assessed with PET, Brain, 1121 (Pt 5) (1998) 931-947.

12) Melzack, R., Psychologic aspects of pain, Res. Publ. Assoc. Res. Nerv. Ment. Dis., 58 (1980) 143-154.

13) Peyron, R., Laurent, B. and Garcia-Larrea, L., Functional imaging of brain responses to pain. A review and meta-analysis (2000), Neurophysiol. Clin., 30 (2000) 263-288.

14) Ploghaus, A., Narain, C., Beckmann, C.F., Clare, S., Bantick, S., Wise, R., et al., Exacerbation of pain by anxiety is associated with activity in a hippocampal network, J. Neurosci., 21 (2001) 9896-9903.

15) Price, C.J. and Friston, K.J., Cognitive conjunction: a new approach to brain activation experiments, Neuroimage, 5 (1997) 261-270.

16) Reiman, E.M., Fusselman, M.J., Fox, P.T., Raichle, M.E., Neuroanatomical correlates of anticipatory anxiety, Science, 243 (1989) 1071-1074.
17) Saab, C.Y. and Willis, W.D., Nociceptive visceral stimulation modulates the activity of cerebellar Purkinje cells, Exp. Brain Res., 140 (2001) 122-126.

18) Svensson, P., Minoshima, S., Beydoun, A., Morrow, T.J. and Casey, K.L., Cerebral processing of acute skin and muscle pain in humans, J. Neurophysiol., 78 (1997) 450-460.

19) Talairach, J. and Tournoux, P., Co-Planar Stereotaxic Atlas of the Human Brain, New York: Thime Medical Publishers, 1988.

20) Talbot, J.D., Marrett, S., Evans, A.C., Meyer, E., Bushnell, M.C. and Duncan, G.H., Multiple representations of pain in human cerebral cortex, Science, 251 (1991) 1355-1358.

21) Yamamoto, T. and Kato, T., Paradoxical correlation between signal in functional magnetic resonance imaging and deoxygenated haemoglobin content in capillaries: a new theoretical explanation, Phys. Med. Biol., 47 (2002) 1121-1141.

Address for correspondence: Takahiro Ushida, M.D.

Department of Orthopaedic Surgery, Kochi Medical School

Kohasu, Okoh-cho, Nankoku, Kochi 783-8505, Japan

Tel.: +81-88-880-2387 Fax: +81-88-880-2388 